Revue de l'Institut des langues et cultures

d'Europe, Amérique, Afrique, Asie et Australie

$34 \mid 2019$

Femmes et migrations auX $\mathrm{XIX}^{\mathrm{e}}$ et $\mathrm{XX} \mathrm{X}^{\mathrm{e}}$ siècles :

regards et représentations

\title{
Immigration féminine cubaine aux États-Unis depuis le début de la Révolution : un phénomène spécifique?
}

Cuban Female Immigration in the United States since the Beginning of the Revolution: A Specific Phenomenon?

Janice Argaillot

(2) OpenEdition

Journals

Édition électronique

URL : http://journals.openedition.org/ilcea/5750

DOI : 10.4000/ilcea.5750

ISSN : 2101-0609

Éditeur

UGA Éditions/Université Grenoble Alpes

Édition imprimée

ISBN : 978-2-37747-074-7

ISSN : $1639-6073$

\section{Référence électronique}

Janice Argaillot, «Immigration féminine cubaine aux États-Unis depuis le début de la Révolution : un phénomène spécifique ? », ILCEA [En ligne], 34 | 2019, mis en ligne le 15 janvier 2019, consulté le 01 mai 2019. URL : http://journals.openedition.org/ilcea/5750 ; DOI : 10.4000/ilcea.5750

Ce document a été généré automatiquement le 1 mai 2019.

(C) ILCEA 


\title{
Immigration féminine cubaine aux États-Unis depuis le début de la Révolution : un phénomène spécifique?
}

\author{
Cuban Female Immigration in the United States since the Beginning of the \\ Revolution: A Specific Phenomenon?
}

Janice Argaillot

\section{Introduction}

1 Les États-Unis représentent depuis plusieurs décennies une terre d'accueil pour les Latino-Américains, même si tous n'y sont pas reçus de la même façon. Ainsi, les relations pour le moins spécifiques entre Cuba et le voisin du Nord, malgré les tentatives de dégel récentes, font de la migration cubaine vers les États-Unis un objet d'étude «à part ».

2 En parallèle, soulignons dès à présent qu'au niveau mondial, la proportion de femmes parmi les migrants a considérablement augmenté ; il s'agit d'un fait important puisque la femme migrante n'a pas nécessairement le même comportement et les mêmes attentes que l'homme migrant, que ce soit vis-à-vis de son pays d'origine ou de sa terre d'accueil. Malgré tout, la présence des femmes dans les mouvements migratoires depuis Cuba vers les États-Unis semble bien peu étudiée (FNUAP, 2006 : 21). Il faut alors s'interroger sur les raisons qui poussent les spécialistes de la question à ne pas tendre vers des analyses mettant en avant les éventuelles différences entre les migrations féminines et masculines. Dans notre cas, nous souhaitons tout particulièrement centrer notre regard sur les femmes cubaines.

3 Ainsi, nous évoquerons dans un premier temps les causes des migrations, qui ne forment pas un tout monolithique, et ne sont pas uniquement liées à une opposition au système politique cubain. Par la suite, nous nous interrogerons sur les modalités et spécificités de 
l'émigration féminine cubaine aux États-Unis, car les études se sont jusqu'à présent concentrées sur les particularismes de l'immigration cubaine aux États-Unis dans son ensemble (on consultera par exemple l'article «Gusanos, Marielitos, Balseros ou Cubanoamericanos: réflexions sur la question migratoire cubaine", de Lorraine Karnoouh). Enfin, nous étudierons les conséquences d'une telle migration, particulièrement sur le plan culturel et identitaire, et nous nous demanderons si le rôle de la femme n'est pas celui d'un pont entre la terre de départ et celle d'arrivée.

\section{Les causes de la migration}

4 Il est sans doute nécessaire de procéder à un bref rappel des principaux mouvements migratoires de Cubains vers les États-Unis depuis le triomphe de la Révolution en 1959, car les diverses vagues migratoires ont été constituées de populations différentes, et ont été différemment accueillies par les États-Uniens. Ainsi, à la question de savoir si l'on peut réellement évoquer " "la" communauté cubaine, entendue comme une communauté soudée et indivise, [nous répondrons qu']en réalité, il existe une stratification interne, liée aux différentes vagues d'immigration et à leur arrivée sur le sol américain » (Capone, 2005 : 139). Dès la fin du xix ${ }^{e}$ siècle, les Cubains sont présents aux États-Unis, puisque la guerre d'indépendance hispano-américaine de 1898 avait fait du voisin du Nord le principal partenaire économique de l'île. Les premiers foyers de l'émigration cubaine se concentrèrent « dans les colonies de la Floride où l'on parlait espagnol, à savoir Miami, Tampa et Key West » (ibid. : 139-140). Si les mouvements migratoires depuis et vers Cuba furent longtemps liés à la problématique du travail, «après 1930, l'incidence de la crise mondiale se traduit par un tarissement du flux, tandis que la révolution castriste [nous dirons cubaine ${ }^{1}$ ] suscit[e] un [nouveau] courant d'émigration [...] » (Charbit, $1987: 129$ ).

5 Pour nous centrer à présent sur la période étudiée dans notre article, soulignons que la première vague d'émigration post-Révolution fut constituée de riches propriétaires ou hommes d'affaires opposés à la Révolution cubaine et qui, pour beaucoup, avaient été expropriés par le gouvernement révolutionnaire. Elle « a été appelée “l'Exil doré”, car elle était formée par les membres des élites cubaines qui avaient tout perdu sur le plan matériel, mais qui possédaient un très important bagage en matière d'éducation et de relations. On estime à 215000 les Cubains qui arrivèrent jusqu'en 1962, année de la crise de la Baie des Cochons qui vit l'arrêt de l'émigration vers les États-Unis » (Capone, $2005: 139$ ). La réforme agraire et les nationalisations de la première étape révolutionnaire poussèrent donc à l'immigration de familles entières, même s'il faut préciser que «l'opération Peter Pan » (1960-1962), menée par les États-Unis - et qui fut un plan de propagande durant lequel on diffusa l'idée que les autorités révolutionnaires allaient abolir l'autorité parentale dans l'île-, provoqua également le départ de 140000 enfants et adolescents, qui quittèrent Cuba sans leur famille (Núñez Sarmiento, 2007). Plus tard, «les vols de la liberté » virent 340000 Cubains quitter leur terre d'origine, de 1965 à 1973. Ces vols, encadrés par les autorités, permettaient aux Cubains qui avaient de la famille aux États-Unis de sortir du pays. Parmi eux, « on remarquait plus de commerçants, de femmes, de personnes âgées, de Juifs et de Chinois, toujours très peu de Noirs » (Rouhaud, 2015).

En 1980, l'exil de Mariel - un port situé à une quarantaine de kilomètres à l'ouest de La Havane - changea encore la donne. Effectivement, les marielitos étaient pour 
la plupart des ouvriers et $40 \%$ d'entre eux étaient considérés comme des Noirs². Cela, ajouté aux rumeurs faisant état dans leurs rangs de la présence de marginaux, de fous et de délinquants, alimenta les préjugés des Américains à leur égard, en net contraste avec l'accueil qui avait été réservé à leurs prédécesseurs, «blancs » et membres des élites économiques et politiques de l'île (Capone, 2005 : 139-140).

En 1994, eut lieu la « crise des balseros » (nom donné aux Cubains qui quittent l'île sur des embarcations de fortune, «balsa » signifiant radeau), qui pour la plus grande partie, et comme pour les marielitos, ne possédaient pas de diplôme universitaire ou de qualifications techniques approfondies. Entre le 13 août et le 13 septembre 1994, 36000 Cubains abandonnèrent l'île, et la plupart étaient des hommes (Delgado Vázquez, 2010).

Nous avons donc dit que le regard porté par les États-Uniens sur les Cubains arrivant aux États-Unis depuis 1959 avait changé, mais il nous semble, comme le souligne Capone, que

la principale différence entre les premières vagues d'immigration et les plus récentes réside dans la perception que leurs membres ont de leur propre statut. Les premiers arrivés ne se sont jamais considérés comme des migrants, mais comme des exilés, dont le seul but était de rentrer à Cuba, une fois Castro tombé (2005: 139-140).

Considérés et se considérant comme des « réfugiés " subissant un départ forcé et un exil traumatique, ils ont été accueillis comme des combattants de l'ennemi communiste, à l'inverse des générations de l'exil de Mariel et des balseros, perçus comme problématiques car incapables, de par leur niveau d'éducation, de s'intégrer dans la société d'accueil et de la faire évoluer « favorablement ».

10 Après ce rappel, et pour en venir à présent aux femmes, on peut dire que dans un premier temps, un grand nombre d'entre elles appuyèrent la Révolution, convaincues par son programme alliant réforme agraire, amélioration de l'habitat, démocratisation de l'accès aux soins et à l'éducation. En outre, le gouvernement révolutionnaire accentua, particulièrement dans les années 1970 , la lutte contre les discriminations envers les femmes (avec par exemple la promulgation du Code de la famille en 1972). En somme, la vague d'émigration plus «politique» des années 1960-1962 fut surtout constituée d'hommes « chefs de famille » qui emmenaient leurs femmes et leurs enfants avec eux, et le départ des femmes ne se fit donc pas de leur propre volonté.

11 Par ailleurs, assez tôt dans la Révolution, le statut de la femme a été bousculé. Certaines évolutions - déclin de la mortalité infanto-juvénile, baisse du coût de l'enfant [durant le temps où Cuba connaissait] un quasi-emploi masculin, système de protection sociale très développé, élévation du niveau d'instruction (Charbit, 1987 :9) - ont incité les femmes cubaines à rester dans l'île. Néanmoins, sur les 50300 Cubains qui ont quitté Cuba entre 1985 et 1992, presque la moitié (47\%) étaient des femmes (Holgado Fernández, 2000 : 306). On constate donc que les évolutions sociales et sociétales n'ont pas permis très longtemps de contrecarrer une émigration motivée par des données politicoéconomiques.

12 Par suite, la perte de l'allié soviétique a débouché sur une crise globale appelée «Période spéciale en temps de paix». Durant les années 1990, de nombreuses pénuries (alimentaires, de combustible ou encore de médicaments) frappèrent l'île, et des coupures d'électricité furent même organisées par le gouvernement afin d'économiser l'énergie. La Période spéciale est donc caractérisée par une émigration avant tout économique, les pénuries liées à l'embargo touchent particulièrement les femmes, en première ligne lorsqu'il s'agit de trouver des denrées pour nourrir la famille - puisque la femme est 
toujours perçue comme la mère nourricière dans bien des familles cubaines -, et affectent a fortiori les femmes enceintes, particulièrement touchées par le manque de médicaments.

13 Ainsi, de nos jours, et malgré les efforts indéniables du gouvernement cubain, la femme a encore à Cuba un rôle d'épouse et de mère avant tout, et le statut de travailleuse, de femme active hors du foyer, n'est pas encore un concept clair dans toutes les familles. En 1970 déjà, seuls 18,5\% de la population économiquement active à Cuba étaient des femmes. Aux États-Unis, à la même date, le taux de femmes cubaines travaillant hors du domicile s'élevait à 47 \% (De Aragón, 1997 : 33). Cela étant, la Période spéciale a dans une certaine mesure reconfiguré la famille cubaine, de nombreux hommes au chômage s'étant vus dans l'obligation de participer beaucoup plus activement aux tâches ménagères ou à l'éducation des enfants. De la sorte, la parité et l'égalité homme-femme sont aujourd'hui défendues par les femmes mais également de plus en plus par les hommes à Cuba - si le gouvernement a très tôt défendu l'égalité hommes-femmes, tous les hommes n'étaient pas prêts à l'accepter et la Période spéciale a sans aucun doute entraîné une prise de conscience générale de la valeur du travail non rémunéré qui incombait aux femmes.

Dans un autre domaine, précisons que de nos jours, les voyages organisés par des passeurs concernent majoritairement les femmes, enfants et familles nucléaires (Delgado Vázquez, 2010), autrement dit fondées sur un couple. En effet, le fait que la traversée soit " organisée » par un tiers habitué au parcours tranquillise les femmes, qui pensent qu'elle sera moins dangereuse. D'une certaine façon, lorsqu'elles quittent l'île en famille, les femmes cubaines conservent durant le voyage vers les États-Unis un rôle d'épouse et de mère, anges gardiens de la famille, veillant sur tous et préoccupées du sort des leurs plus que de leur propre devenir.

15 Ainsi, les femmes cubaines sont devenues de véritables protagonistes de la migration, puisque la Oficina Nacional de Estadística e Información (ONEI) estime sur son site internet qu'entre 2002 et 2012, 263000 Cubaines ont quitté l'île, temporairement ou définitivement. En 2010-2011, on constatait que plus de femmes que d'hommes émigraient depuis Cuba. Sur 1250600 émigrés, 640800 étaient des femmes, et 609800 des hommes. La migration cubaine aux États-Unis était ainsi constituée de femmes à 50,6 \% (OCDE, $2015:$ 144), femmes qui connaissaient un taux de chômage de 9,6 \% (ibid. : 145). La tendance semble s'inverser depuis très peu de temps, avec une progression de la migration masculine depuis Cuba vers les États-Unis. Ces variations, et les liens complexes entre le "Crocodile vert ${ }^{3}$ » et le voisin du Nord ne sont cependant pas les seules spécificités de la migration féminine cubaine vers les États-Unis.

\section{Spécificités et caractéristiques de la migration féminine cubaine vers les États-Unis}

Concernant ce point, une première question s'impose: pourquoi les études sur les mouvements migratoires cubains n'incluent-elles que très rarement la dimension du genre ? Cela est d'autant plus étonnant que les Cubaines ne lient pas les mêmes relations avec leur terre d'arrivée que d'autres migrantes latino-américaines:

Les travaux sur les immigrantes cubaines [...] ne correspondent ni au paradigme classique émancipation contre oppression, ni aux stéréotypes raciaux, ni à ceux de sous-classe sociale. Au contraire, la recherche note, chez les Cubains en général et chez les Cubaines en particulier, une aptitude aux progrès économiques et à 
l'assimilation culturelle. Les immigrantes cubaines sont plus proches de la classe moyenne, dans les domaines social et économique, que leurs consœurs d'Amérique latine : du coup, dans la société comme dans la sphère économique américaine, elles sont plus mobiles que les Mexicaines, que les Dominicaines ou que les femmes de Porto Rico. La recherche reflète aussi le thème de l'exil, qui continue à définir la communauté cubaine des États-Unis. L'exil étant habituellement considéré comme le rejet d'une personne par son État-nation d'origine (plus que comme le rejet hors des traditions et des institutions sociales et culturelles du pays d'accueil), la dimension de genre est secondaire dans les études sur l'exil. (Schneider, 2003)

L'exil et la migration n'étant pas perçus de la même façon dans les inconscients et imaginaires collectifs, on se concentre dans de nombreuses études sur le rejet d'un système politique que symbolise le départ de Cuba, et sur le rejet des exilés par «la communauté nationale» - que l'on considère être celle qui vit sur le territoire national -, autrement dit, sur le déchirement entre la communauté cubaine « de l'île » et celle « de l'exil », instaurant ainsi un clivage profond au sein de la population cubaine. La dimension genrée passe alors au second plan, mais un « point de vue transversal de genre s'avère utile en tant qu'outil théorique et méthodologique indispensable pour étudier les changements provoqués par l'émigration dans les relations de pouvoir et subordination au sein de la famille " (Delgado Vázquez, 2010). En effet, pour celui qui part, celui qui reste, et même dans le cas d'un départ impliquant le couple ou la famille, on constate que le déplacement opère un changement de position ou induit une redéfinition des statuts entre homme et femme.

18 Cette redéfinition peut passer par l'insertion des migrantes cubaines sur le marché de l'emploi états-unien. En 1979, c'est-à-dire à un moment où l'exil doré s'était installé et n'avait pas été rejoint par les marielitos ou les balseros, les femmes cubaines de plus de 15 ans vivant aux États-Unis avaient un revenu moyen de 8982 US\$, alors que la moyenne pour les autres femmes hispaniques s'élevait à 8923 US\$, et celle des femmes étatsuniennes à 10380 US\$. Les femmes cubaines gagnaient moins que les hommes (14 168 US \$), mais légèrement plus que les autres femmes immigrées (De Aragón, 1997 : 33). Faut-il voir dans cette donnée la force de travail plus importante chez les Cubaines présentée par certaines études? Il faut surtout dire que les conditions d'accueil aux États-Unis sont bien différentes pour les Cubains et les autres communautés. En effet, les lois en vigueur stipulent que tous les Cubains arrivant aux États-Unis (et quelle que soit la façon dont ils y arrivent) peuvent demander l'asile politique. La Ley de ajuste cubano (instaurée en novembre 1966) prévoit même que tout Cubain ayant séjourné un an sur le sol états-unien peut devenir automatiquement citoyen des États-Unis. Les accords migratoires de 1994-1995 ont quant à eux débouché sur la politique des "pieds secs, pieds mouillés ». L'administration Clinton avait ainsi décidé que les Cubains interceptés en mer seraient renvoyés directement sur le sol cubain, tandis que ceux ayant atteint les États-Unis seraient autorisés à demander la citoyenneté états-unienne. Cette loi a été abrogée par le gouvernement de Barack Obama juste avant la fin de son mandat. La distinction entre communautés s'opère également dans certains questionnaires remis aux immigrés vivant aux États-Unis (et notamment ceux du bureau de recensement), puisqu'on observe que les autorités font une différence entre " origine hispanique » et " races noire ou asiatique ». Les termes ont leur importance, et le fossé induit par le mot « race » ne se retrouve pas dans «l'origine hispanique». On a plutôt l'impression que cette origine, bien que distincte de l'anglo-saxonne, n'est pas totalement honteuse. 
Quoi qu'il en soit, « les soldes migratoires par sexe entre 1960 et 2003 démontrent que le nombre de femmes a été supérieur à celui des hommes en chiffres absolus sur l'ensemble de ces quarante-trois années. Quant aux soldes migratoires par année, à 25 reprises, le nombre de femmes a dépassé celui des hommes " (Núñez Sarmiento, 2007). On estimait en 2010 que les migrants cubains vers les États-Unis étaient principalement des femmes, majoritairement jeunes et possédant un niveau de qualification médian ou élevé. Le niveau d'études différencie d'ailleurs les femmes cubaines d'autres groupes de migrantes, et peuvent leur permettre une insertion plus rapide dans le monde du travail - même si beaucoup obtiennent des emplois sur le territoire d'arrivée pour lesquels elles sont surqualifiées.

La sociologue Marta Núñez, dans une étude réalisée en 2009, montrait la forte hausse de la proportion de femmes jeunes et seules parmi les migrants cubains, femmes qui, pour la plupart, fondaient une famille sur la terre d'arrivée. Pour d'autres chercheurs, cette décision est pour partie liée au rôle de mère assignée par la société cubaine :

Beaucoup de femmes qui décident d'émigrer le font seules - [ou plutôt] non accompagnées par un conjoint, puisque beaucoup partent avec leurs enfants - et dans le but premier d'aider économiquement la famille qu'elles laissent à Cuba. Cette situation marque une différence qualitative en termes migratoires, en présentant la femme comme actrice, protagoniste du mouvement, non dépendante d'une quelconque figure masculine, comme peuvent l'être l'époux, le père ou le frère. Quant à la décision de partir seules ou avec leur descendance, on pourrait analyser plusieurs facteurs. Ainsi, l'autorisation de l'autre parent pour que l'enfant sorte de Cuba, indispensable selon la loi cubaine, n'est pas toujours accordée par le père, situation qui oblige la femme à émigrer seule pour ensuite tenter une réunification familiale ou abandonner son fils ou sa fille. Un autre aspect qui a pu influer sur la décision de certaines femmes d'émigrer seules est la pratique cubaine, en vigueur jusqu'en janvier 2013, d'autoriser seulement la sortie des mineurs pour une émigration définitive, et non temporaire. (Alfonso Martínez, 2014 : 51)

21 Le fait de priver leur enfant d'un retour possible et «facile» à Cuba conduit donc certaines femmes à ne pas les emmener avec elles - ce qui montre bien un attachement à la terre d'origine, et une volonté d'améliorer une situation économique plus que de fuir un système politique. Nous l'avons dit, les femmes cubaines et plus largement caribéennes étant encore principalement en charge de l'éducation des enfants, et dans bien des cas, du soin aux personnes âgées et malades de la famille, il leur est sans doute plus difficile de prendre la décision d'émigrer. En outre, une fois cette décision prise, les impacts sur la cellule familiale et sur les membres de la famille, même élargie, sont multiples.

Pour les femmes de la première vague de 1960, pour lesquelles la migration s'était effectuée en famille, le travail hors du foyer a été possible, afin de contribuer au maintien du niveau de vie dont la famille bénéficiait lorsqu'elle vivait à Cuba. Il s'agissait donc plus d'une obligation que d'un déshonneur pour le mari censé subvenir aux besoins du ménage. Mais les tâches domestiques sont restées "typiquement» féminines, et l'autorité, en particulier vis-à-vis des enfants, incombait toujours au père (De Aragón, 1997 :35). Néanmoins, il semble impossible d'éviter un certain syncrétisme, et on constate que les femmes cubaines vivant aux États-Unis tendent à s'émanciper de leurs maris et à s'affirmer comme les véritables décisionnaires de leurs vies (ibid.). Si l'influence des États-Unis dans l'île a bien longtemps été rejetée, car perçue comme une menace de " coca-colarisation ", l'influence positive de la culture états-unienne de parité pour une partie des femmes cubaines vivant aux États-Unis ne semble donc plus à démontrer. 
23 À l'inverse, un autre point intéressant est celui de la proportion croissante de «Latinos » aux États-Unis, et de leur place toujours plus importante dans les domaines économique et politique, qui impliquent sans doute un bouleversement pour l'identité états-unienne. Malgré tout, au déchirement de la migration s'ajoute l'exclusion dont peuvent être victimes les Cubains à leur arrivée aux États-Unis. L'entraide, voire le communautarisme, est alors une nécessité, et d'ailleurs, «la Petite Havane est plus qu'un espace physique, elle représente un rêve, un substitut de La Havane et un référent identitaire pour tout Cubain vivant aux États-Unis » (Rouhaud, 2015). La langue espagnole est officiellement la deuxième langue dans l'État de New York, en Californie et en Floride. D'une façon générale, le taux de Cubains d'origine à Miami montre que cette ville est un lieu d'ancrage pour la communauté cubaine qui quitte lî̂le. Du fait de sa proximité géographique et de son infrastructure, Miami - et son comté - attire en effet les migrants cubains : « Lieu d'ancrage des migrants caribéens (notamment des Cubains, des Haïtiens, des Nicaraguayens), sa population hispano-américaine a été multipliée par 20 entre 1960 et 1990 et représente aujourd'hui plus du tiers de sa population totale, soit plus de 700000 personnes. » (Hartog, 2006 : 173) Si nous ne souhaitons pas revenir sur la « cubanisation » de Miami ${ }^{4}$ ou « l'hispanisation » de certains États, nous souhaitons comprendre le rôle de la femme cubaine dans l'éventuelle recréation d'un espace identitaire sur le sol étatsunien.

Peut-être de façon surprenante, la femme cubaine, par sa volonté de "réussir » sur le territoire d'arrivée, a largement contribué à faire de Miami une ville «latinoaméricaine » :

L'hispanisation de Miami va de pair avec son expansion économique. Dans cette réussite, il faut souligner le rôle des femmes cubaines qui participèrent au travail salarié même si elles étaient femmes au foyer à Cuba. Pendant que les grandsparents gardaient les enfants (modèle du ménage multi générations), elles travaillaient dans des blanchisseries, ateliers de couture, cantinas pour servir à domicile des repas cubains. Pourtant cette contribution n'a pas été suffisamment mise en valeur. (Rouhaud, 2015)

Ce qui interroge plus particulièrement ici, c'est le fait que le rôle mais également le ressenti de ces femmes aient été laissés de côté, et n'aient pas fait l'objet d'analyses scientifiques plus précises. Les Cubaines éprouvent bien sûr le déchirement, le tiraillement entre le " je », le " nous », le « ils », et la difficulté qu'ont tous les migrants à se définir ou à se sentir « chez eux » quelque part. De nombreux artistes et écrivains ont fait part de leur sentiment d'être finalement toujours dans un entre-deux ne permettant pas une autodéfinition claire. C'est alors le passage d'une communauté migrante à une communauté diasporique qui se joue :

Le concept de diaspora s'emploie de plus en plus. Certains auteurs l'associent à l'idée de dispersion (l'acte de quitter le lieu d'origine) et d'autres en font un synonyme de communautés transnationales. Quoi qu'il en soit, il existe un consensus sur le fait que le concept se réfère à une construction sociale de groupes ethniques minoritaires d'origine étrangère ou d'une collectivité transnationale déterritorialisée, qui ont une identité commune, qui résident dans des pays récepteurs, mais maintiennent un très fort lien sentimental et matériel, réel ou imaginaire, avec leur terre natale et reconnaissent, en outre, le reflet de celle-ci dans leurs pratiques idiomatiques, religieuses et culturelles. (Martínez Pizarro, 2003 : 16-17)

26 La femme, dans la déterritorialisation et le déracinement, reste le pilier de la culture d'origine, tout en devenant une charnière avec la culture de la terre d'accueil. On observe 
effectivement un autre changement du rôle de celle-ci au sein de la famille. Au début, la femme était celle qui se préoccupait de l'intégration des enfants dans la société d'accueil par l'apprentissage de l'anglais et l'éducation; aujourd'hui, elle a également un rôle de transmission de la culture originelle, à travers la langue espagnole et la littérature hispanique, les fêtes et rites religieux ou les contes traditionnels pour enfants du pays que l'on a quitté (De Aragón, 1997 : 37). L'enfance se construit donc avec le pays quitté en «fond », en arrière-plan, qui, s'il peut être idéalisé, n'est en tout cas pas caché. Loin de constituer un tabou, il devient un référent identitaire dont on se montre fier. Ainsi, " pour tout groupe en exil le lieu où il tente de s'enraciner est un marqueur identitaire fort, comme la langue, la race et l'histoire » (Rouhaud, 2015), ce qui signifie que le lieu que l'on a quitté (ou que les ancêtres ont quitté) est également ancré dans l'identité du migrant et de ses descendants.

Malgré tout, le lien entre territoire d'origine et d'arrivée ne semble pas être strictement le même pour les hommes et les femmes, puisque «moins de femmes que d'hommes veulent rentrer à Cuba. Dans le même temps, les femmes, plus que les hommes, défendent le droit d'envoyer des médicaments, de la nourriture, de voyager librement à et depuis Cuba » (De Aragón, 1997 : 38). On en déduit que les femmes ayant quitté l'île se sentent assez bien sur le territoire d'arrivée pour y rester, et souhaitent sans doute pour beaucoup inscrire le soutien financier qu'elles offrent à leur famille dans la durée ; elles veulent également avoir la possibilité de retourner à Cuba temporairement, et qu'il soit permis à leurs enfants de retourner définitivement, ou pour de longs laps de temps, dans l'île. L'homme se perçoit plus comme un immigré « de passage ", souhaitant améliorer durant quelque temps sa situation économique et celle des siens, mais désirant bien souvent vivre ses vieux jours à Cuba. Cette différence de point de vue, cette manière bien distincte d'envisager la sortie du territoire ne peut conduire qu'à des conséquences distinctes de la migration pour les hommes et les femmes.

\section{Entre perceptions et représentations de la femme cubaine aux États-Unis}

Durant de nombreuses années, le gouvernement cubain a mis en scène la sortie des " exilés », qui sont devenus des boucs émissaires, des victimes expiatoires, permettant à ceux qui restaient de se défouler. À titre d'exemple, les images diffusées par la télévision cubaine lors de l'exil de Mariel «montrent le cordon de policiers qui retient une foule amassée sur le port, une foule dont le seul objectif est d'insulter et de malmener ceux qui ont choisi l'exil. " Vers de terre! Chiennes! Traitres! Fils de pute! », peut-on entendre. Le cordon policier est brisé. Des hommes et des femmes attaquent à coups de bâtons et de pierres les postulants à l'exil, vieillards, enfants et femmes enceintes. Des gens en larmes, d'autres le visage en sang... Et que dit le journaliste de la chaine de télévision cubaine? « La milice et la police ont du mal à contenir la colère du peuple contre les traîtres à leur patrie. » (Manet, 2004)

Mais l'évolution des causes provoquant le départ a entraîné une évolution du regard porté par les Cubains de Cuba sur ceux qui partent. Ainsi, la plupart des migrantes cubaines qui ont quitté l'île depuis le début des années 1990 ont gardé un contact avec leurs familles; elles leur envoient souvent de l'argent («remesas»), et l'assouplissement récent des conditions de voyage leur permet de se rendre à Cuba afin de visiter leurs 
proches. Elles sont bien considérées comme des immigrées économiques par ces derniers, et non des exilées politiques. Elles symbolisent la nécessité (terme employé par de nombreux Cubains que j'ai pu interroger à ce sujet) pour les familles « d'envoyer » un de leurs membres à l'extérieur afin de subsister. Le départ n'est donc pas toujours synonyme de fuite discrète ou clandestine, il est parfois et au contraire concerté en famille, le membre désigné pour partir étant celui qui semble le plus à même de bien réussir économiquement sur le territoire d'arrivée. Dès lors, le contact entre la femme migrante et sa famille se fait sans qu'intervienne un jugement de valeur négatif sur son départ - on peut même dire que beaucoup de familles ont réellement besoin de l'aide apportée par leurs membres vivant à l'extérieur, et présentent donc ces derniers comme des victimes de l'oppression états-unienne plus que comme des dommages collatéraux d'un système politique en déroute.

30 Si l'aide apportée par l'envoi d'argent ou de présents est reconnue et valorisée par les Cubains de l'île, alors qu'elle a été perçue par certains aux États-Unis comme une façon de provoquer un soulèvement de la population cubaine (Martínez Pizarro, 2003:17), le départ des proches est malgré tout souvent vécu comme un abandon, et l'absence de certains membres de la famille peut même déstabiliser cette dernière. On ne peut nier une certaine perte de repères pour les enfants, surtout ceux qui sont élevés par leurs grands-parents du fait de l'absence de leurs parents. Si vivre à trois générations sous le même toit est monnaie courante à Cuba, le fait qu'il en manque une provoque un vide, et un questionnement chez les enfants qui pour certains se demandent pourquoi leurs parents ne les ont pas emmenés avec eux (bien souvent, parce qu'ils étaient trop jeunes pour affronter la traversée, ou pouvaient constituer un obstacle à l'installation sur la nouvelle terre).

31 Par ailleurs, les problèmes de communication liés à l'embargo - car la reprise des relations entre Cuba et les États-Unis n'en est qu'à ses balbutiements - font que «le départ d'un membre de la famille, en particulier dans le cas de couples, signifie très souvent la fin de la relation. Les contacts transnationaux sont également moins importants chez les ressortissants cubains, mais cela s'explique en partie par la situation politique entre Cuba et les États-Unis » (Mallet, 2014). Par ailleurs, et « dans l'ensemble, là où les Latinos d'Amérique latine voient généralement leur immigration comme un sacrifice pour le bien de la famille, les Latinos caribéens considèrent plutôt l'immigration comme la quête individuelle d'une meilleure qualité de vie » (ibid.). On constate donc que de nos jours, l'émigration se fait de façon plus «individualiste " chez les Caribéens. Cependant, les Cubaines qui quittent l'île sont, nous le répétons, et d'après différentes études de grandes travailleuses, qui travaillent avant tout pour le bien de leurs familles, faisant passer leur propre bien-être ou leur réalisation personnelle au second plan. Sans aucune intention de les déifier, il faut dire que les Cubaines apparaissent réellement comme des migrantes " hors normes ». "L'éthique du travail de la famille, plus que les réussites individuelles, a été [selon le $\mathrm{D}^{\mathrm{r}}$ Lisandro Pérez] la clé de la réussite cubaine. Pérez signale que les femmes cubaines travaillent plus, non seulement que les femmes des autres groupes hispaniques, mais aussi que les Nord-Américaines. »(De Aragón, 1997 : 32)

Mais il faut encore souligner que les discriminations existent au sein même de la communauté cubaine vivant aux États-Unis. Les «anciens " peuvent ainsi adopter des attitudes négatives face aux nouveaux arrivants, ces derniers accaparant dans l'esprit des premiers des postes et aides qui leur revenaient. En outre, des jalousies entre les différentes communautés latinos existent : 
Certains des facteurs qui entravent la solidarité entre groupes latinos peuvent avoir pour origine le traitement particulier dont font l'objet certains groupes nationaux comme les Cubains et les Portoricains ${ }^{5}$. Leur statut confère à ces deux groupes nationaux certains privilèges dont ne bénéficient pas d'autres Latinos, ce qui est à l'origine de tensions entre les communautés de Latinos, tensions suscitées par le ressentiment des Latinos non-cubains et non-portoricains. (Mallet, 2014)

$\mathrm{Au}$ cours des entretiens menés par la chercheuse Marie Mallet, "les mots "jaloux", "sentiment de supériorité/d'infériorité", "injuste" et "abus" sont ressortis de manière répétée pour caractériser le traitement différencié dont bénéficient ces deux catégories » (ibid.). Rappelons que l'immigration latino-américaine est composée de différents groupes latinos, et que "Miami compte une très large communauté cubaine, tandis qu'à Los Angeles les Mexicains constituent la majeure partie des Latinos et que les Portoricains et les Dominicains sont prédominants à Boston » (ibid.).

On peut également évoquer le cas emblématique d'Elián Sanchez, et de sa mère. En 1999, Elián et sa mère avaient quitté clandestinement Cuba, accompagnés d'autres Cubains, sur un radeau. L'embarcation de fortune avait chaviré, et la mère du garçonnet l'avait sauvé en le hissant sur une chambre à air avant de se noyer. Une fois arrivé à Miami, Elian avait été recueilli par un grand-oncle, qui se disait prêt à l'élever. Mais d'un autre côté, son père appelait de ses vœux son retour dans l'île. Aux États-Unis, la communauté cubaine était divisée sur la question. En fin de compte, on a vu dans « l'affaire Elián » l'abnégation dont toute mère doit faire preuve vis-à-vis de ses enfants. La femme cubaine a alors été présentée comme un être sacrifié et se sacrifiant, mais trahissant tout de même son pays. La symbolique forte a contribué à faire de l'enfant un pantin, l'objet d'un combat politicoidéologique.

D'ailleurs, l'influence politique des Cubains aux États-Unis est bien souvent mise en avant dans les médias. Notons tout de même que les sénateurs Marco Rubio et Ted Cruz, qui ont été candidats à l'investiture républicaine lors de la campagne présidentielle de 2016, sont des hommes et que la seule figure qui semble se distinguer est celle d'Ileana Ros-Lehtinen, née à La Havane en 1952 ; elle est membre du Parti républicain et représente la Floride à la Chambre des représentants depuis presque 30 ans ; depuis 2011, elle est également présidente du Comité des affaires étrangères de la même chambre. Cette faible représentation de la femme d'origine cubaine est toutefois comparable à celle de femmes venues d'autres pays, et est similaire à la situation des États-Uniennes, même s'il semble que la « cubanité » soit un problème s'ajoutant à celui de la féminité :

De même que pour les Nord-Américaines, les Cubaines se cognent à un plafond de verre (glass ceiling) dans le monde de l'entreprise, qu'il leur est impossible de briser. Si ce n'est au sein de leurs propres entreprises, très peu de femmes parviennent à de hautes fonctions de direction. [Du fait de] certaines structures patriarcales cubaines, les Cubaines ne sont pas représentées dans les comités de direction des associations civiques et politiques de la diaspora, même s'il y a naturellement des exceptions qui confirment la règle. Il suffit de regarder les journaux. Vous verrez des photos de femmes membres d'associations de charité (le canon de l'ange qui se déplace de l'espace privé à l'espace public) et celles d'hommes impliqués dans les activités économiques et politiques. La présence féminine parmi les « dirigeants de l'exil », à l'exception du domaine de la lutte pour les droits de l'homme, a été et est toujours nulle. (De Aragón, 1997 : 37)

En 1990, les Cubaines résidant aux États-Unis (nées ou non à Cuba) travaillaient principalement dans les secteurs de la vente, de l'administration et des services (ibid. : 34). Ces emplois relativement « traditionnels » et souvent peu payés ne permettent donc pas à la femme cubaine de s'émanciper totalement et d'atteindre de hautes fonctions 
politiques ou professionnelles. Celle-ci demeure toujours une citoyenne de seconde zone, ou tout au moins reléguée au second plan et que l'on ne met en valeur que lors de meetings électoraux à des fins de propagande.

On peut enfin dire que des associations telles que le "Cuban Women's Club", fondées en 1969, sont avant tout des coalitions de femmes hispanoaméricaines «dont les buts montrent une grande acculturation (leurs objectifs sous-jacents: "networking», " empowering other women", sont difficilement traduisibles parce qu'ils portent en eux des concepts culturels états-uniens actuels) » (ibid. : 37). Malgré tout, le Cuban Women's Club, auquel les femmes peuvent adhérer quelle que soit leur nationalité, a abandonné dans les années 1970 ses activités "de charité " pour se tourner vers des conférences et manifestations ayant pour thème le rôle de ses membres dans la société, le bilinguisme, etc., c'est-à-dire des préoccupations en phase avec celles des immigrées cubaines.

\section{Conclusion}

Les femmes ne manquent ni de détermination ni d'ingéniosité lorsqu'elles décident de quitter le territoire cubain. À titre d'exemple, en mai 2015, la première femme à réussir seule la traversée entre Cuba et les États-Unis sur un radeau accostait en Floride. Elle était en outre partie de Santiago, effectuant là un long voyage ( 2 semaines et 3 jours). En 2004, une Cubaine s'était envoyée par colis postal aux États-Unis. Cela pose évidemment la question de la raison profonde du départ: mettre sa vie en jeu dans une traversée incertaine démontre en effet une volonté de fuir des difficultés (matérielles notamment) qui ne sont plus à démontrer. Ainsi, si l'on ne peut que rappeler que les relations migratoires entre Cuba et les États-Unis reflètent les difficiles relations qu'ont entretenues, et qu'entretiennent encore les deux pays, il faut insister sur l'indifférenciation des études menées jusqu'alors sur les êtres humains qui composent la migration. Ces relations montrent bien le peu de cas que l'on fait de la femme, y compris dans l'étude de phénomènes aussi déterminants que les mouvements de population. On ne peut que le regretter lorsque l'on voit combien la femme migrante influe sur la recréation d'un espace identitaire original dans le pays d'arrivée, agissant comme un trait d'union culturel entre deux pays que tout semble opposer, et comme une charnière depuis le passé vers le futur de leurs familles, qui s'établiront ou non durablement sur le sol états-unien.

Si les causes du départ des femmes depuis Cuba vers les États-Unis sont autant politiques qu'économiques, il faut rappeler que leurs modalités diffèrent souvent des migrations masculines, et qu'elles restent dépendantes de considérations avant tout familiales. Enfin, les femmes cubaines qui quittent lî̂le lui font bien sûr défaut, mais contribuent dans le même temps à créer plus qu'à recréer un nouvel espace identitaire de l'autre côté du détroit de Floride, ouvrant alors un nouveau monde des possibles. 


\section{BIBLIOGRAPHIE}

ALFONSO MARTíNEZ María Isabel (2014), Las migraciones internacionales: especial referencia a las mujeres migrantes cubanas (mémoire de master), Université d'Oviedo, <http:// dspace.sheol.uniovi.es/dspace/bitstream/10651/27495/6/TFM_Alfonso\%20Martinez.pdf> (8 juin 2015).

ARAGÓN Uva DE (1997), « La mujer cubana en Estados Unidos. Algunas consideraciones sobre su aporte socioeconómico y las modificaciones de su papel », Annual Meeting of the Association for the Study of the Cuban Economy, 7, 32-39, <www.ascecuba.org/c/wp-content/uploads/2014/09/v07Aragon.pdf> (8 juin 2015).

CAPONE Stefania (2005), Les Yoruba du nouveau monde. Religion ethnicité et nationalisme noir aux ÉtatsUnis, Paris : Karthala.

CHARBIT Yves (1987), Famille et nuptialité dans la Caraibe, Paris : Institut national d'études démographiques.

Delgado VÁzquez Denisse (2010), « Impacto en la dinámica familiar de la emigración de algunos de sus miembros. Un estudio de caso en el Consejo Popular "El Carmelo" ", Novedades en Población, 7(14), 256-273, <www.novpob.uh.cu/index.php/NovPob/article/download/175/208>

(22 novembre 2015).

FNUAP - FONDS DES NATIONS UNIES POUR LA POPULATION (2006), État de la population mondiale 2006. Vers l'espoir : les femmes et la migration internationale, New York : FNUAP.

HARTOG Thierry (2006), «Quelques éléments d'identité spatiale caribéenne : entre singularité et altérité », P. Joseph (dir.), La Caraibe, données environnementales, Paris : Karthala, 167-178.

HOLGADO FERNÁNDEZ Isabel (2000), ¡No es fácil ! Mujeres cubanas y la crisis revolucionaria, Barcelone : Icaria.

KARNOOUH Lorraine (2007), « Gusanos, Marielitos, Balseros ou Cubanoamericanos : réflexions sur la question migratoire cubaine », A. Calmont \& C. Audebert, Dynamiques migratoires de la Caraibe, Paris : Karthala, 63-76.

MALLET Marie L. (2014), « Les communautés latinos aux USA. L'impossibilité d'un label panethnique », en ligne sur La Vie des idées : <www.laviedesidees.fr/Les-communautes-latinos-auxUSA.html> (8 juin 2015).

MANET Eduardo (2004), Rhapsodie cubaine, Paris : Grasset.

MARTíneZ PIZARRo Jorge (2003), El mapa migratorio de América Latina y el Caribe, las mujeres y el género , Santiago du Chili : CEPAL.

NÚÑEZ SARMIENTO Marta (2007), « Género y migraciones externas en Cuba entre 1985 y 2005: resultados preliminares », Novedades en población, 3(6), 45-70, <www.novpob.uh.cu/index.php/ NovPob/article/download/103/136> (8 juin 2015).

ORGANISATION DE COOPÉRATION ET DÉVELOPPEMENT ÉCONOMIQUES (2015), Resserrer les liens avec les diasporas. Panorama des compétences de migrants 2015, Paris : Éditions OCDE. 
RouHAUd Adeline (2015), « La reconstruction du lieu d'origine. L'exemple de la Pequeña Habana à Miami », Cahiers d'études des cultures ibériques et latino-américaines, 1, 121-135, <http:// cecil.upv.univ-montp3.fr/files/2015/12/CECIL_1_2015_8_ar.pdf> (16 décembre 2015).

SCHNEIDER Dorothée (2003), «L'immigration féminine aux États-Unis : un essai historiographique ", Actes de l'histoire de l'immigration, 3, <http://barthes.ens.fr/clio/revues/AHI/ articles/volumes/wo.html> (8 juin 2015).

\section{NOTES}

1. Car la révolution n'est pas celle de Fidel ou de Raúl Castro, elle est celle du peuple cubain.

2. Deux catégories s'imposaient, les « Noirs» s'opposant aux «Blancs», comme si Cuba n'avait connu aucun métissage, et les « Noirs » se voyant largement discriminés.

3. L'un des surnoms de Cuba.

4. Le comté de Miami Dade regroupe $59 \%$ des migrants cubains.

5. Terme problématique, Porto Rico n'étant pas une nation indépendante, mais qui montre sans doute combien la construction identitaire peut se faire hors des cadres politiques imposés.

\section{RÉSUMÉS}

Dès les prémices de la Révolution, les autorités cubaines ont mis l'accent, dans leurs discours et dans les lois, sur la lutte contre les discriminations de genre. Malgré tout, la société cubaine ne s'est pas départie de certains clichés sexistes, et le rôle des femmes en son sein demeure ambivalent. Ainsi, si elle est présentée comme l'un des piliers de la Révolution, la femme cubaine est également l'étai de nombreuses familles, à tous les niveaux. Dès lors, depuis 1959, son ancrage au territoire cubain ou au contraire son départ n'ont pas été sans conséquences pour l'île et l'ensemble de sa population.

Pour différentes raisons qu'il conviendra d'analyser, les flux migratoires depuis Cuba se sont principalement orientés vers « le voisin du Nord », et nous nous proposons tout d'abord d'étudier plus particulièrement les causes et principales périodes d'émigration féminine depuis Cuba vers les États-Unis de 1959 à nos jours. Dans un deuxième temps, notre travail s'intéressera aux spécificités des migrations féminines cubaines vers les États-Unis, et nous nous demanderons notamment si ces migrations entraînent la recréation d'un espace culturel et identitaire, en cherchant à savoir quel peut être le rôle spécifique de la femme cubaine dans l'appropriation du nouveau territoire. Enfin, nous aborderons les multiples impacts de cette migration, au travers du regard porté sur les femmes cubaines aux États-Unis tant par leur communauté d'origine que par leur communauté d'accueil. En effet, il conviendra de s'interroger sur le poids des migrantes cubaines dans les systèmes politiques et économiques cubain et états-unien, et nous pourrons alors nous demander si Miami n'est pas désormais une nouvelle frontière cubaine.

Since the beginning of the Revolution, Cuban authorities have emphasized the fight against sexual discrimination in their speeches and in the law. However, Cuban society has not departed from some sexist clichés and the part played by women within it remains ambivalent. Indeed, while they are presented as one of the pillars of the Revolution, Cuban women are also the main 
support in many families, at every level. As a consequence, since 1959, their integration into the Cuban territory or, conversely, their departure from it, has impacted both the Island and its population.

For different reasons-which this paper will analyze-, migration flows from Cuba have been primarily directed to "the Northern Neighbor". We will therefore first study more particularly the reasons for this emigration as well as the main periods of female emigration since 1959. In a second part, this work will focus on the specificities of Cuban women's migrations to the United States, and we will examine whether these migrations have led to the re-creation of a cultural and identity space and what specific role Cuban woman may have played in the appropriation of the new territory. Lastly, this paper will dwell on the multiple impacts of this migration by analyzing how Cuban women are perceived in the United States, both by their original community and their host community. The influence of cuban female migrants on both the Cuban and American political and economic systems will be looked into, and we shall then wonder whether Miami may or not be considered a new Cuban border.

\section{INDEX}

Mots-clés : Cuba, Révolution, États-Unis, femmes, migrations

Keywords : Cuba, Revolution, United States, women, migrations

\section{AUTEUR}

\section{JANICE ARGAILLOT}

Université Grenoble Alpes, ILCEA4.

Janice Argaillot est docteur en études latino-américaines (Université de Cergy-Pontoise, 2011), maître de conférences en civilisation latino-américaine et langue espagnole de spécialité (Université Grenoble Alpes) depuis septembre 2013.

Membre du CERHIUS-ILCEA4 et du GRIAHAL, elle travaille principalement sur les relations culturelles cubano-caribéennes, Cuba et sa société depuis le début de la Révolution cubaine, la culture cubaine et ses manifestations. Auteur de plusieurs articles dans des revues spécialisées, elle communique également de façon régulière sur ces sujets dans des colloques internationaux. 